\title{
MATURAÇÃO E QUALIDADE PÓS-COLHEITA DE AMEIXAS 'LAETITIA' COM A APLICAÇÃO PRÉ-COLHEITA DE AVG e GA ${ }_{3}^{1}$
}

\author{
CRISTIANO ANDRÉ STEFFENS ${ }^{2}$, CASSANDRO VIDAL TALAMINI DO AMARANTE ${ }^{3}$, \\ RICARDO CHECHI ${ }^{4}$, JOÃO PAULO GENEROSO SILVEIRA ${ }^{5}$ E THAIS ROSELI CORRÊA ${ }^{5}$
}

RESUMO - O objetivo deste trabalho foi avaliar o efeito da aplicação pré-colheita de aminoetoxivinilglicina (AVG; $\left.0 ; 90 ; 125 \mathrm{mg} \mathrm{L}^{-1}\right)$ e ácido giberélico $\left(\mathrm{GA}_{3} ; 0\right.$ e $\left.100 \mathrm{mg} \mathrm{L}^{-1}\right)$ sobre a maturação e a qualidade de ameixas 'Laetitia' após o armazenamento refrigerado. Foi utilizado o delineamento em blocos casualizados, com seis tratamentos (três doses de AVG x duas doses de $\mathrm{GA}_{3}$ ) e quatro repetições. A aplicação do $\mathrm{GA}_{3} \mathrm{e}$ do AVG foi realizada 28 e 7 dias antes do início da primeira colheita, respectivamente. O experimento foi conduzido em 2007 e em 2009. Em cada ano foram realizadas três colheitas, sendo 11-01-2007, 17-01-2007 e 25-01-2007 (colheita comercial); 15-01-2009, 22-01-2009 (colheita comercial), e 29-01-2009. Em 2007, frutos da terceira colheita foram armazenados em uma câmara fria comercial durante 22 dias, a $0,5^{\circ} \mathrm{C}$, e umidade relativa de $92 \%$ e mais cinco dias de exposição em condição ambiente (temperatura média de $23^{\circ} \mathrm{C}$ e umidade relativa média de $60 \%)$. A aplicação pré-colheita de AVG $\left(125 \mathrm{mg} \mathrm{L}^{-1}\right)$, isoladamente ou $\mathrm{GA}_{3}$, ou $\mathrm{GA}_{3}+\mathrm{AVG}$, retarda a maturação de ameixas 'Laetitia' na planta. O tratamento pré-colheita de ameixas 'Laetitia' com GA ${ }_{3}$, seguido da aplicação de AVG, manteve maior firmeza da polpa dos frutos após o armazenamento refrigerado.

Termos para indexação: Prunus salicina, reguladores de crescimento, etileno.

\section{MATURATION AND POSTHARVEST QUALITY OF 'LAETITIA'PLUMS WITH PREHARVEST TREATMENT OF AVG AND GA}

\begin{abstract}
The objective of this study was to evaluate the effects of preharvest spraying of Japanese plum trees (Prunus salicina, cv. Laetitia) with aminoethoxyvinilglycine [AVG; at 0, 90, and $125 \mathrm{mg} \mathrm{L}^{-1}$ ] and giberellic acid $\left[\mathrm{GA}_{3}\right.$; at 0 and $\left.100 \mathrm{mg} \mathrm{L}^{-1}\right]$ on maturation and fruit quality after cold storage. The experiment followed a randomized block design, with six treatments (three doses of AVG $\mathrm{x}$ two doses of $\mathrm{GA}_{3}$ ) and four replicates. The sprayings with $\mathrm{GA}_{3}$ and AVG were performed at 28 and seven days before first fruit harvesting, respectively. Experiment was carried out during the years of 2007 and 2009. Fruits were harvested 3 times, being 01/11/2007, 01/17/2007 and 01/25/2007 (commercial harvest) 2007, and 01/15/2009, 01/22/2009 (commercial harvest) e 01/29/2009, in 2009. At 2007, fruits of the last harvest were stored in commercial cold storage during 22 days $\left(0.5^{\circ} \mathrm{C} / 92 \% \mathrm{RH}\right)$, followed by five days of shelf life $\left(23^{\circ} \mathrm{C} / 60 \% \mathrm{RH}\right)$. Delays in harvesting induces of fruit ripening, however preharvest treatment only with AVG $\left(125 \mathrm{mg} \mathrm{L}^{-1}\right)$ singly or $\mathrm{GA}_{3}$, followed or not by the application of AVG, delay fruit ripening of 'Laetitia' plums plant. Preharvest spraying of 'Laetitia' plum trees with $\mathrm{GA}_{3}$ followed by the application of AVG preserves the consistence of the pulp fruit after cold storage.
\end{abstract}

Index terms: Prunus salicina, growth regulators, ethylene.

\footnotetext{
1(Trabalho 069-10). Recebido em: 12-03-2010. Aceito para publicação em: 10-12-2010.

${ }^{2}$ Dr. Prof. do Departamento de Agronomia, CAV/UDESC, Av. Luiz de Camões, 2090, Caixa Postal 281, CEP: 88520-000, Lages-SC. Autor para correspondência. Email: steffens@cav.udesc.br;

${ }^{3} \mathrm{PhD}$., Bolsista de Produtividade em Pesquisa do CNPq, Professor do Departamento de Agronomia, CAV/UDESC, Lages-SC. Email: amarante@cav.udesc.br

${ }^{4}$ Acadêmico do Curso de Agronomia da UDESC, bolsista de iniciação cientifíca, Centro de Ciências Agroveterinárias (CAV), Universidade do Estado de Santa Catarina (UDESC). E-mail: a6rc@cav.udesc.br;

${ }^{5}$ Programa de Pós-Graduação em Ciências Agrárias, Centro de Ciências Agroveterinárias (CAV), Universidade do Estado de Santa Catarina (UDESC). E-mail:, joaop-silveira@hotmail.com, thaisroselicorrea@hotmail.com.
} 


\section{INTRODUÇÃO}

A safra brasileira de ameixas é muito curta, o que favorece a entrada de ameixas importadas para a complementação da oferta sazonal. O armazenamento é uma alternativa viável para estender o período de oferta do produto nacional, mas ainda é pouco praticado. Além disso, a ameixa, devido ao seu metabolismo acelerado, apresenta um período de armazenamento muito curto (BRACKMANN et al., 2005), o que concentra o período de comercialização. Desta forma, poucos dias de atraso na maturação dos frutos na planta ou um aumento no período de armazenamento podem causar um incremento expressivo no preço de venda do produto e aumentar a receita por parte do produtor.

$\mathrm{Na}$ fruticultura, os reguladores de crescimento que inibem a síntese ou ação do etileno vêm sendo utilizados com o intuito de retardar a maturação e preservar a qualidade dos frutos durante o armazenamento, como é o caso da maçã (STEFFENS et al., 2005; AMARANTE; STEFFENS, 2009), caqui (FERRI et al., 2004) e pêssego (AMARANTE et al., 2005), podendo tornar-se uma tecnologia acessível, também aos produtores de ameixas.

O composto aminoetoxivinilglicina (AVG) inibe a síntese do etileno e retarda todos os eventos fisiológicos que são estimulados por este fitormônio, dos quais muitos estão relacionados ao amadurecimento dos frutos. Em maçãs, além de reduzir a queda pré-colheita e atrasar a maturação dos frutos, a aplicação de AVG permite melhor manutenção da qualidade dos frutos durante o armazenamento, pois proporciona frutos mais verdes, com maior firmeza de polpa, maior acidez titulável (AT) e menor quantidade de sólidos solúveis (SS), além de ocorrer menor incidência de podridões e distúrbios fisiológicos (STEFFENS et al., 2005; AMARANTE; STEFFENS, 2009). Ju et al. (1999) observaram que a pulverização de pessegueiros com AVG apresentou efeito significativo no retardo da maturação e do amadurecimento, e na redução da incidência de escurecimento interno da polpa nos frutos. Em ameixas, tem sido verificado que a aplicação de AVG retarda a maturação (STEFFENS et al., 2009).

Outro regulador de crescimento que tem demonstrado efeito sobre o controle da maturação e amadurecimento dos frutos é o ácido giberélico $\left(\mathrm{GA}_{3}\right)$. Alguns trabalhos têm demonstrado que o $\mathrm{GA}_{3}$ retarda a maturação de caqui, reduzindo o amarelecimento e proporcionando maior firmeza de polpa, acidez titulável e peso de frutos, além de reduzir podridões no armazenamento (FERRI et al., 2004). Em ameixas, também foi verificado efeito do $\mathrm{GA}_{3}$ sobre o retardo da maturação (STEFFENS et al., 2009). A aplicação de giberelina em frutos de caqui resultou em uma redução parcial da ação de etileno, retardando o amolecimento, a perda de clorofila e o acúmulo de carotenoides (BRACKMANN et al., 2002). Estes efeitos são usados com o objetivo de retardar a colheita e prolongar o tempo de armazenamento dos frutos (FERRI et al., 2004). A degradação da clorofila, a síntese de pigmentos amarelados e/ ou avermelhados e a redução da firmeza de polpa podem ser parcialmente inibidas com aplicações de $\mathrm{GA}_{3}$ em pré-colheita (BEN-ARIE et al., 1996). Segundo Pech et al. (1994), o GA atua inibindo a ação de clorofilases e a produção de etileno, sendo desconhecido o mecanismo de ação e a forma de atuação nestas vias bioquímicas.

Em pêssegos, Ju et al. (1999) e Amarante et al. (2005) observaram efeito sinérgico entre a AVG e o $\mathrm{GA}_{3}$ no controle da maturação. Assim, tratamentos à base de $\mathrm{AVG}$, em combinação com $\mathrm{GA}_{3}$, representam grande potencial para utilização em pomares comerciais de ameixa 'Laetitia'. Estas duas substâncias, associadas, podem retardar o ponto de colheita e manter a qualidade dos frutos no armazenamento e aumentar o tamanho médio de frutos, permitindo assim maiores ganhos aos produtores. No entanto, ainda não existem informações sobre o efeito conjunto da aplicação pré-colheita de AVG e $\mathrm{GA}_{3}$ sobre a maturação e manutenção da qualidade de ameixas 'Laetitia' após o armazenamento.

O objetivo deste trabalho foi avaliar o efeito da aplicação pré-colheita de AVG e $\mathrm{GA}_{3}$ sobre a maturação e a manutenção da qualidade de ameixas 'Laetitia' após o armazenamento refrigerado.

\section{MATERIAL E MÉTODOS}

Os experimentos foram conduzidos nos anos agrícolas de 2006/2007 e 2008/2009 com ameixeiras da cultivar Laetitia, em pomar comercial, localizado no município de Lages-SC.

Os tratamentos consistiram da pulverização pré-colheita das ameixeiras com AVG (i.a. cloridrato de aviglicina $15 \%$, fabricante: Valente Bioscience Corporation) e $\mathrm{GA}_{3}$ (i.a. ácido giberélico $10 \%$, fabri- 
cante: Abbot Laboratories - Chemical \& Agricultural Products Division), segundo um esquema bifatorial, combinando três doses de AVG (0; 90 e $\left.125 \mathrm{mg} \mathrm{L}^{-1}\right)$ com duas doses de $\mathrm{GA}_{3}\left(0\right.$ e $\left.100 \mathrm{mg} \mathrm{L}^{-1}\right)$. Foi utilizado pulverizador pressurizado com $\mathrm{CO}_{2}$, adicionando-se à calda o espalhante adesivo Silwet ${ }^{\circledR}(0,01 \% \mathrm{v} / \mathrm{v})$, com aplicação até o ponto de escorrimento foliar. $\mathrm{O}$ $\mathrm{GA}_{3}$ e o AVG foram aplicados 28 e sete dias antes da primeira colheita, respectivamente.

O delineamento experimental utilizado foi de blocos ao caso, com quatro repetições. Cada repetição foi constituída por 10 plantas (cada parcela com seis plantas úteis e duas de bordadura em cada extremidade da fileira), sendo que, de cada parcela, foram colhidos 25 frutos aleatoriamente. Foram realizadas três colheitas em cada ano de execução do experimento, sendo 11-01-2007, 17-01-2007 e 25-01-2007(colheita comercial), em 2007, e 15-012009, 22-01-2009 (colheita comercial) e 29-01-2009 em 2009.

Após a colheita, foram realizadas as determinações de atributos de textura (forças para ruptura da epiderme, penetração da polpa e compressão do fruto), AT, SS e cor da epiderme, através do ângulo 'hue' $\left(h^{\circ}\right)$.

Na safra de 2006/2007, também foram colhidos, em 25-01-2007, 25 frutos em cada parcela, que foram armazenados em câmara fria comercial (temperatura de $0,5^{\circ} \mathrm{C}$ e UR de $92 \%$ ) por 22 dias e mais cinco dias de exposição dos frutos em condições ambiente (temperatura média de $23^{\circ} \mathrm{C}$ e umidade relativa média de $60 \%$ ) para simular o período de comercialização. Após esse período, os frutos foram analisados quanto aos seguintes atributos de qualidade: firmeza de polpa, atributos de textura, cor da epiderme, AT, SS e ocorrência de podridões.

A firmeza de polpa (N) foi determinada na região equatorial dos frutos, em dois lados opostos, após remoção de uma pequena porção da epiderme, com o auxílio de um penetrômetro manual equipado com ponteira de 7,9 mm de diâmetro.

Os atributos de textura $(\mathrm{N})$ foram analisados com um texturômetro eletrônico TAXT-plus ${ }^{\circledR}$ (Stable Micro Systems Ltd., Reino Unido), em termos de forças necessárias para a ruptura da epiderme, penetração na polpa e compressão do fruto. Para a quantificação das forças necessárias para a ruptura da epiderme e penetração na polpa, foi utilizada ponteira modelo PS2, com $2 \mathrm{~mm}$ de diâmetro, a qual foi introduzida na polpa a uma profundidade de $5 \mathrm{~mm}$.
A força para compressão do fruto foi determinada usando-se uma ponteira modelo $\mathrm{P} / 75$, com $75 \mathrm{~mm}$ de diâmetro, que exerceu uma força de compressão até uma deformação de $5 \mathrm{~mm}$ na superfície do fruto.

Os valores de AT (meq de ácido cítrico 100 $\mathrm{mL}^{-1}$ ) foram obtidos através de uma amostra de 10 $\mathrm{mL}$ de suco dos frutos, previamente extraído de fatias transversais retiradas da região equatorial das ameixas e trituradas em uma centrífuga elétrica. Esta amostra foi diluída em $90 \mathrm{~mL}$ de água destilada e titulada com solução de hidróxido de sódio $0,1 \mathrm{~N}$ até $\mathrm{pH} 8,1$.

Os teores de SS ( ${ }^{\circ}$ Brix) foram determinados por refratometria, utilizando-se do suco extraído conforme descrito para a acidez titulável, sendo realizada a correção do efeito da temperatura $\left(20^{\circ} \mathrm{C}\right)$.

A determinação da cor da epiderme $\left(h^{\circ}\right)$ foi efetuada com um colorímetro Minolta, modelo CR 400. As leituras foram realizadas na região equatorial, nos lados menos e mais vermelhos dos frutos. $\mathrm{O} \mathrm{h}^{\circ}$ define a coloração básica, sendo que $0^{\circ}=$ vermelho, $90^{\circ}=$ amarelo e $180^{\circ}=$ verde .

A ocorrência de podridões (\%) foi avaliada pela contagem dos frutos afetados, interna e externamente, com lesões maiores do que $5 \mathrm{~mm}$ de diâmetro causadas por agentes patogênicos.

Os dados foram submetidos à análise da variância (ANOVA). Dados em porcentagem foram transformados através da fórmula arcosseno $[(\mathrm{x}+0,5) / 100]^{1 / 2}$ antes de serem submetidos à ANOVA. Para a comparação das médias, adotou-se o teste de Tukey $(P<0,05)$.

\section{RESULTADOS E DISCUSSÃO}

\section{Maturação}

Não foi verificado interação entre tratamentos e as data de colheita para todas as variáveis, em ambos os anos agrícolas (Tabelas 1; 2; 3 e 4). Este mesmo comportamento foi observado em pêssego 'Rubidoux' (AMARANTE et al., 2005). Desta forma, apenas os dados médios dos atributos avaliados, referentes às três épocas de colheita de cada tratamento, foram submetidos à análise estatística.

$\mathrm{O}$ uso do $\mathrm{AVG}$ e do $\mathrm{GA}_{3}$, isoladamente ou em combinação, proporcionou maiores valores de forças para a ruptura da casca, penetração na polpa e compressão do fruto, em 2007 (Tabela 1). Em 2009, estes reguladores de crescimento também 
apresentaram efeito na manutenção dos atributos de textura, exceto o AVG, na dose de $90 \mathrm{mg} \mathrm{L}^{-1}$ para forças para penetração da polpa e compressão do fruto (Tabela 2). Outros trabalhos também verificaram maior consistência da polpa em maçãs tratadas com AVG (STEFFENS et al., 2006; AMARANTE; STEFFENS, 2009) e em cerejas (CANLI; ORHAN, 2009) e pêssegos (JU et al., 1999; AMARANTE et al., 2005) tratados com GA G $_{3}$ Em pêssegos, o uso de $\mathrm{GA}_{3}$ e AVG apresentou efeito sinérgico na manutenção da firmeza de polpa (JU et al., 1999; AMARANTE et al., 2005). A resposta da textura dos frutos aos tratamentos com AVG está diretamente relacionada ao seu efeito sobre a redução na síntese do etileno. O etileno é necessário para a promoção na atividade de enzimas responsáveis pela redução da firmeza dos frutos (MAJUMDER; MAZUMDAR, 2002). Majumder e Mazumdar (2002) constataram que o aumento na atividade da enzima poligalacturonase foi altamente correlacionado à evolução na concentração de etileno. Ben-Arie et al. (1996) afirmam que o $\mathrm{GA}_{3}$ mantém a consistência dos frutos por retardar ou inibir as mudanças estruturais na parede celular que acompanham o amolecimento dos frutos. $\mathrm{O}$ mecanismo de ação do $\mathrm{GA}_{3}$ sobre a manutenção da integridade da estrutura da parede celular e, consequentemente, da textura dos frutos, também parece estar relacionado ao seu efeito na redução da produção de etileno, reduzindo assim a atividade da enzimas hidrolíticas da parede celular (PECH et al., 1994).

Em ambos os anos agrícolas, a força para ruptura da casca apresentou os maiores valores nos tratamentos $\mathrm{GA}_{3}$ e $\mathrm{GA}_{3}+\operatorname{AVG}\left(125 \mathrm{mg} \mathrm{L}^{-1}\right)$, que não diferiram do tratamento $\mathrm{GA}_{3}+\mathrm{AVG}\left(90 \mathrm{mg} \mathrm{L}^{-1}\right)$ (Tabelas 1 e 2). Similarmente, Amarante et al. (2005) observaram melhor efeito do $\mathrm{GA}_{3}$, aplicado isoladamente ou seguido da aplicação com AVG, sobre a manutenção da firmeza da polpa em pêssego 'Rubidoux', do que a aplicação isolada de AVG.

No ano de 2007, os tratamentos $\mathrm{GA}_{3}+$ AVG (125 $\mathrm{mg} \mathrm{L}^{-1}$ ) retardou a evolução da coloração da epiderme (maior valor de $h^{\circ}$ ), em relação ao tratamento-controle (Tabela 3). No ano de 2009, os tratamentos AVG (125 mg L-1), GA $\mathrm{mg}_{3}+\mathrm{AVG}$ (90 e $125 \mathrm{mg} \mathrm{L}^{-1}$ ) mantiveram os frutos mais verdes, em relação ao tratamento-controle, contudo os melhores resultados foram obtidos nos frutos tratados com $\mathrm{GA}_{3}$, independentemente da aplicação de AVG (Tabela 4). Como a mudança na cor durante o ama- durecimento de ameixas é um processo dependente de etileno (ALVES et al., 2009; 2010), isto explica a menor intensidade de coloração vermelha nos tratamentos com $\mathrm{GA}_{3}$ e AVG. Em caqui e pêssego, também foi observado retardo na evolução da coloração da epiderme em resposta à aplicação de $\mathrm{GA}_{3}$, devido a seu efeito no retardo da maturação (FERRI et al., 2004; AMARANTE et al., 2005). Amarante et al. (2005) também observaram melhores resultados na manutenção da coloração da epiderme de pêssego 'Rubidoux' com a aplicação pré-colheita de $\mathrm{GA}_{3}$, independentemente da aplicação de AVG.

A AT não apresentou diferenças entre tratamentos, em ambos os anos (Tabelas 3 e 4). Em maçãs 'Gala', a aplicação pré-colheita de AVG também não influenciou na AT dos frutos (STEFFENS et al., 2006; AMARANTE; STEFFENS, 2009). Ferri et al. (2004), trabalhando com caqui 'Fuyu', também não observaram efeito significativo do tratamento com $\mathrm{GA}_{3}$ sobre a manutenção da AT, corroborando os resultados obtidos no presente trabalho. Steffens et al. (2005, 2006), ao não observarem efeito da aplicação de AVG na manutenção da AT em maçãs 'Gala', afirmaram que, possivelmente, o processo de degradação dos ácidos pode não ser grandemente influenciado pelo etileno. Contudo, em pêssego 'Rubidoux', a aplicação de GA, combinada com AVG, resultou em frutos com maior AT (AMARANTE et al., 2005).

Os teores de SS, em 2007, foram menores nos frutos tratados com AVG, $\mathrm{GA}_{3}$ e $\mathrm{GA}_{3}+\mathrm{AVG}$, do que naqueles do tratamento-controle. Dentre os tratamentos com reguladores de crescimento, os frutos tratados com $\mathrm{GA}_{3}$ e $\mathrm{GA}_{3}+\mathrm{AVG}\left(90\right.$ e $\left.125 \mathrm{mg} \mathrm{L}^{-1}\right)$ apresentaram os menores valores de SS (Tabela 3 ). No ano de 2009, o único tratamento que não diferiu do controle foi o AVG na dose de $90 \mathrm{mg} \mathrm{L}^{-1}$, sendo que os valores mais baixos de $\mathrm{SS}$ foram observados nos tratamentos com $\mathrm{GA}_{3}$, independentemente da aplicação de AVG (Tabela 4). De maneira geral, os teores de SS em pêssegos 'Rubidoux' também foram menores nos frutos de plantas pulverizadas com $\mathrm{GA}_{3}$, independentemente da aplicação de AVG (AMARANTE et al., 2005). Possivelmente, os maiores valores de $\mathrm{SS}$ no tratamento-controle estejam relacionados ao maior conteúdo de pectinas solúveis, uma vez que estes frutos apresentaram a menor firmeza de polpa. Relação inversa entre valores de firmeza de polpa e SS já foi observada em ameixa 'Laetitia' (STEFFENS et al., 2009).

Os diferentes atributos de textura apresenta- 
ram alterações semelhantes em resposta à aplicação dos tratamentos (Tabelas 1 e 2). As alterações dos diferentes atributos de textura avaliadas decorrem da degradação da parede das células dos frutos, processo que é induzido pelo etileno. Todavia, segundo Guillermin et al. (2006), pode haver diferenças entre essas variáveis.

O atraso na colheita dos frutos causou redução nos atributos de textura, da AT e dos teores de SS, bem como resultou na evolução da coloração vermelha da epiderme dos frutos, em ambos os anos de avaliação (Tabelas $1 ; 2 ; 3$ e 4). Isto é considerado um comportamento normal durante o amadurecimento dos frutos (STEFFENS et al., 2006; STEFFENS et al., 2009).

A aplicação isolada de AVG, considerando os valores de textura, firmeza de polpa e cor da epiderme, permitiu um período de retardo na colheita inferior a sete dias. $\mathrm{O} \mathrm{GA}_{3}$, isolado ou em associação com o AVG, pode permitir um atraso na colheita de 7 a 10 dias. Um atraso na colheita de 7 dias pode ser muito desejável para a ameixa 'Laetitia', uma vez que o período de colheita é extremamente curto. Todavia, o AVG, na cultura da macieira, apresenta um período de carência de 28 dias, porém, em ameixas, não há informações sobre o período de carência desta substância. Já para o $\mathrm{GA}_{3}$, não há intervalo de segurança, pois é de ocorrência natural em plantas. Considerando os melhores resultados do $\mathrm{GA}_{3}$ e que não possui carência, sua utilização pode ser a alternativa mais interessante para o retardo do amadurecimento de ameixas 'Laetitia'.

\section{Qualidade pós-colheita}

Após 22 dias de armazenamento refrigerado mais cinco dias em condição ambiente, a firmeza de polpa e as forças para a penetração da polpa e para a compressão do fruto apresentaram maiores valores nos frutos dos tratamentos com $\mathrm{GA}_{3}\left(100 \mathrm{mg} \mathrm{L}^{-1}\right)$ seguido da aplicação com AVG (90 e $\left.125 \mathrm{mg} \mathrm{L}^{-1}\right)$, para frutos colhidos no dia 25-01-07 (Tabela 5). Ju et al. (1999) e Amarante et al. (2005) também observaram, após o armazenamento refrigerado, melhor manutenção da consistência da polpa em pêssegos tratados com $\mathrm{GA}_{3}$ seguido pela aplicação com AVG. A resposta da consistência de polpa ao $\mathrm{GA}_{3}$ combinado com o AVG, após o armazenamento, está diretamente relacionada ao seu efeito sobre a redução na síntese de etileno (AMARANTE et al., 2005). Conforme citado anteriormente, o etileno é necessário para a promoção na atividade de enzimas responsáveis pela perda de firmeza de polpa (MAJUMDER; MAZUMDAR, 2002). Embora na colheita não tenha sido avaliada a firmeza de polpa dos frutos, através dos dados de textura da polpa (forças para a penetração da polpa e compressão do fruto), pôde-se verificar que a aplicação pré-colheita isolada de $\mathrm{GA}_{3}$ ou $\mathrm{AVG}$, apesar de retardarem a perda de consistência da polpa na colheita, não mantiveram o mesmo efeito após o armazenamento.

Os teores de SS foram maiores nos frutos do tratamento-controle, que não diferiu do tratamento AVG (90 $\mathrm{mg} \mathrm{L}^{-1}$ ) (Tabela 5). Possivelmente, os maiores valores de sólidos solúveis nestes frutos estejam relacionados ao maior conteúdo de pectinas solúveis, uma vez que estes frutos apresentaram os menores valores de firmeza de polpa e de forças para penetração da polpa e compressão do fruto. Relação inversa entre valores de firmeza de polpa e teores de SS também foi observada em ameixas 'Laetitia' por Steffens et al. (2009).

As variáveis: força para ruptura da epiderme, AT, cor da epiderme e incidência de podridões, não apresentaram diferenças entre tratamentos, após 22 dias de armazenamento refrigerado seguido por cinco dias em condição ambiente (dados não apresentados). 
TABELA 1 - Atributos de textura em ameixas 'Laetitia', na colheita, em função da aplicação pré-colheita de aminoetoxivinilglicina (AVG) e ácido giberélico $\left(\mathrm{GA}_{3}\right)$ e data de colheita, no ano de 2007.

\begin{tabular}{|c|c|c|c|c|}
\hline \multirow[b]{2}{*}{ Tratamentos } & \multicolumn{3}{|c|}{$2006 / 2007$} & \multirow[b]{2}{*}{ Média } \\
\hline & $1^{\text {a }}$ Colheita & $2^{\text {a }}$ Colheita & $3^{\text {a }}$ Colheita & \\
\hline \multicolumn{5}{|c|}{ Força para a penetração da polpa $(\mathrm{N})$} \\
\hline Controle & 3,46 & 2,92 & 2,45 & $2,95 c^{*}$ \\
\hline $\operatorname{AVG}\left(90 \mathrm{mg} \mathrm{L}^{-1}\right)$ & 3,57 & 3,23 & 2,65 & $3,15 b$ \\
\hline $\operatorname{AVG}\left(125 \mathrm{mg} \mathrm{L}^{-1}\right)$ & 3,72 & 3,18 & 2,64 & $3,18 b$ \\
\hline $\mathrm{GA}_{3}\left(100 \mathrm{mg} \mathrm{L}^{-1}\right)$ & 3,89 & 3,83 & 2,63 & $3,45 \mathrm{a}$ \\
\hline $\mathrm{GA}_{3}\left(100 \mathrm{mg} \mathrm{L}^{-1}\right)+\operatorname{AVG}\left(90 \mathrm{mg} \mathrm{L}^{-1}\right)$ & 3,77 & 3,78 & 2,56 & $3,37 \mathrm{ab}$ \\
\hline $\mathrm{GA}_{3}^{3}\left(100 \mathrm{mg} \mathrm{L}^{-1}\right)+\operatorname{AVG}\left(125 \mathrm{mg} \mathrm{L}^{-1}\right)$ & 3,82 & 3,75 & 2,84 & $3,46 \mathrm{a}$ \\
\hline Média & $3,74 \mathrm{~A}$ & $3,48 \mathrm{~B}$ & $2,66 \mathrm{C}$ & \\
\hline \multirow[t]{2}{*}{$\mathrm{CV}(\%)$} & \multicolumn{3}{|c|}{6,64} & \\
\hline & \multicolumn{3}{|c|}{ Força para a ruptura da epiderme $(\mathrm{N})$} & Média \\
\hline Controle & 11,2 & 11,0 & 10,5 & $10,9 b$ \\
\hline AVG (90 mg L-1) & 11,8 & 11,4 & 12,1 & $11,8 \mathrm{a}$ \\
\hline $\operatorname{AVG}\left(125 \mathrm{mg} \mathrm{L}^{-1}\right)$ & 11,8 & 11,4 & 11,0 & $11,4 \mathrm{a}$ \\
\hline $\mathrm{GA}_{3}\left(100 \mathrm{mg} \mathrm{L}^{-1}\right)$ & 12,2 & 12,6 & 10,4 & $11,7 \mathrm{a}$ \\
\hline $\mathrm{GA}_{3}\left(100 \mathrm{mg} \mathrm{L}^{-1}\right)+\operatorname{AVG}\left(90 \mathrm{mg} \mathrm{L}^{-1}\right)$ & 12,2 & 12,2 & 11,2 & $11,8 \mathrm{a}$ \\
\hline $\mathrm{GA}_{3}\left(100 \mathrm{mg} \mathrm{L}^{-1}\right)+\operatorname{AVG}\left(125 \mathrm{mg} \mathrm{L}^{-1}\right)$ & 12,2 & 12,2 & 10,8 & $11,7 \mathrm{a}$ \\
\hline Média & $11,9 \mathrm{~A}$ & $11,8 \mathrm{~A}$ & $11,0 \mathrm{~B}$ & \\
\hline \multirow[t]{2}{*}{ CV $(\%)$} & \multicolumn{3}{|c|}{5,68} & \\
\hline & \multicolumn{3}{|c|}{ Força para a compressão do fruto (N) } & Média \\
\hline Controle & 123,5 & 100,1 & 77,6 & $100,4 \mathrm{~b}$ \\
\hline $\operatorname{AVG}\left(90 \mathrm{mg} \mathrm{L}^{-1}\right)$ & 132,8 & 114,6 & 90,3 & $112,6 a$ \\
\hline $\operatorname{AVG}\left(125 \mathrm{mg} \mathrm{L}^{-1}\right)$ & 133,2 & 108,7 & 95,7 & $112,5 \mathrm{a}$ \\
\hline $\mathrm{GA}_{3}\left(100 \mathrm{mg} \mathrm{L}^{-1}\right)$ & 136,9 & 124,8 & 85,9 & $115,9 \mathrm{a}$ \\
\hline $\mathrm{GA}_{3}\left(100 \mathrm{mg} \mathrm{L}^{-1}\right)+\operatorname{AVG}\left(90 \mathrm{mg} \mathrm{L}^{-1}\right)$ & 135,3 & 127,0 & 91,1 & $117,8 \mathrm{a}$ \\
\hline $\mathrm{GA}_{3}\left(100 \mathrm{mg} \mathrm{L}^{-1}\right)+\operatorname{AVG}\left(125 \mathrm{mg} \mathrm{L}^{-1}\right)$ & 132,7 & 129,9 & 82,2 & $114,9 \mathrm{a}$ \\
\hline Média & $132,4 \mathrm{~A}$ & $117,5 \mathrm{~B}$ & $87,1 \mathrm{C}$ & \\
\hline $\mathrm{CV}(\%)$ & & 9,73 & & \\
\hline
\end{tabular}

* Médias seguidas por mesma letra, maiúscula na horizontal e minúscula na vertical, não diferem entre si, pelo teste de Tukey $(\mathrm{p}<0,05)$. 
TABELA 2 - Atributos de textura em ameixas 'Laetitia', na colheita, em função da aplicação pré-colheita de aminoetoxivinilglicina (AVG) e ácido giberélico $\left(\mathrm{GA}_{3}\right)$ e data de colheita, no ano de 2009.

\begin{tabular}{|c|c|c|c|c|}
\hline \multirow[b]{2}{*}{ Tratamentos } & \multicolumn{3}{|c|}{$2008 / 2009$} & \multirow[b]{2}{*}{ Média } \\
\hline & $1^{\text {a }}$ Colheita & $2^{\text {a }}$ Colheita & $3^{\text {a }}$ Colheita & \\
\hline \multicolumn{5}{|c|}{ Força para a penetração da polpa (N) } \\
\hline Controle & 3,39 & 2,86 & 2,40 & $2,88 \mathrm{c}^{*}$ \\
\hline AVG $\left(90 \mathrm{mg} \mathrm{L}^{-1}\right)$ & 3,39 & 3,09 & 2,57 & $3,02 b$ \\
\hline $\operatorname{AVG}\left(125 \mathrm{mg} \mathrm{L}^{-1}\right)$ & 3,63 & 3,10 & 2,57 & $3,10 \mathrm{~b}$ \\
\hline $\mathrm{GA}_{3}\left(100 \mathrm{mg} \mathrm{L}^{-1}\right)$ & 4,10 & 4,04 & 2,74 & $3,63 \mathrm{a}$ \\
\hline $\mathrm{GA}_{3}\left(100 \mathrm{mg} \mathrm{L}^{-1}\right)+\operatorname{AVG}\left(90 \mathrm{mg} \mathrm{L}^{-1}\right)$ & 3,81 & 3,82 & 2,59 & $3,41 \mathrm{ab}$ \\
\hline $\mathrm{GA}_{3}\left(100 \mathrm{mg} \mathrm{L}^{-1}\right)+\operatorname{AVG}\left(125 \mathrm{mg} \mathrm{L}^{-1}\right)$ & 4,16 & 4,09 & 3,10 & $3,78 \mathrm{a}$ \\
\hline Média & $3,75 \mathrm{~A}$ & $3,50 \mathrm{~B}$ & $2,66 \mathrm{C}$ & \\
\hline \multirow[t]{2}{*}{ CV $(\%)$} & \multicolumn{3}{|c|}{$\begin{array}{c}8,39 \\
\end{array}$} & \\
\hline & Força pa & ruptura da el & erme $(\mathrm{N})$ & Média \\
\hline Controle & 10,98 & 10,78 & 10,29 & $10,68 b$ \\
\hline $\operatorname{AVG}\left(90 \mathrm{mg} \mathrm{L}^{-1}\right)$ & 10,62 & 10,26 & 10,89 & $10,59 b$ \\
\hline $\operatorname{AVG}\left(125 \mathrm{mg} \mathrm{L}^{-1}\right)$ & 11,51 & 11,12 & 10,73 & $11,12 \mathrm{a}$ \\
\hline $\mathrm{GA}_{3}\left(100 \mathrm{mg} \mathrm{L}^{-1}\right)$ & 13,18 & 13,61 & 11,23 & $12,67 \mathrm{a}$ \\
\hline $\mathrm{GA}_{3}\left(100 \mathrm{mg} \mathrm{L}^{-1}\right)+\operatorname{AVG}\left(90 \mathrm{mg} \mathrm{L}^{-1}\right)$ & 12,32 & 12,32 & 11,31 & $11,99 \mathrm{a}$ \\
\hline $\mathrm{GA}_{3}\left(100 \mathrm{mg} \mathrm{L}^{-1}\right)+\operatorname{AVG}\left(125 \mathrm{mg} \mathrm{L}^{-1}\right)$ & 13,30 & 13,30 & 11,77 & $12,79 \mathrm{a}$ \\
\hline Média & $11,98 \mathrm{~A}$ & $11,90 \mathrm{~A}$ & $11,04 \mathrm{~B}$ & \\
\hline \multirow[t]{2}{*}{ CV $(\%)$} & & 7,99 & & \\
\hline & \multicolumn{3}{|c|}{ Força para a compressão do fruto $(\mathrm{N})$} & Média \\
\hline Controle & 121,03 & 98,10 & 76,05 & $98,39 b$ \\
\hline $\operatorname{AVG}\left(90 \mathrm{mg} \mathrm{L}^{-1}\right)$ & 119,52 & 103,14 & 81,27 & $101,31 b$ \\
\hline $\operatorname{AVG}\left(125 \mathrm{mg} \mathrm{L}^{-1}\right)$ & 129,87 & 115,90 & 93,31 & $113,03 \mathrm{a}$ \\
\hline $\mathrm{GA}_{3}\left(100 \mathrm{mg} \mathrm{L}^{-1}\right)$ & 137,85 & 126,78 & 92,77 & $119,13 \mathrm{a}$ \\
\hline $\mathrm{GA}_{3}\left(100 \mathrm{mg} \mathrm{L}^{-1}\right)+\operatorname{AVG}\left(90 \mathrm{mg} \mathrm{L}^{-1}\right)$ & 136,65 & 128,27 & 92,01 & $118,98 \mathrm{a}$ \\
\hline $\mathrm{GA}_{3}\left(100 \mathrm{mg} \mathrm{L}^{-1}\right)+\operatorname{AVG}\left(125 \mathrm{mg} \mathrm{L}^{-1}\right)$ & 134,64 & 131,58 & 89,60 & $118,61 \mathrm{a}$ \\
\hline Média & $129,93 \mathrm{~A}$ & $117,29 \mathrm{~B}$ & $87,5 \mathrm{C}$ & \\
\hline $\mathrm{CV}(\%)$ & & 6,86 & & \\
\hline
\end{tabular}

*Médias seguidas por mesma letra, maiúscula na horizontal e minúscula na vertical, não diferem entre si, pelo teste de Tukey (p<0,05). 
TABELA 3 -Cor da epiderme, acidez titulável e sólidos solúveis em ameixas 'Laetitia', na colheita, em função da aplicação pré-colheita de aminoetoxivinilglicina (AVG) e ácido giberélico $\left(\mathrm{GA}_{3}\right) \mathrm{e}$ data de colheita, no ano de 2007.

\begin{tabular}{|c|c|c|c|c|}
\hline \multirow[b]{2}{*}{ Tratamentos } & \multicolumn{3}{|c|}{$2006 / 2007$} & \multirow[b]{2}{*}{ Média } \\
\hline & $1^{\text {a }}$ Colheita & $2^{\text {a }}$ Colheita & $3^{\text {a }}$ Colheita & \\
\hline \multicolumn{5}{|c|}{ Cor (hue) } \\
\hline Controle & 78,2 & 73,7 & 65,6 & $72,5 b^{*}$ \\
\hline $\operatorname{AVG}\left(90 \mathrm{mg} \mathrm{L}^{-1}\right)$ & 83,9 & 86,9 & 71,0 & $80,6 \mathrm{ab}$ \\
\hline $\operatorname{AVG}\left(125 \mathrm{mg} \mathrm{L}^{-1}\right)$ & 82,7 & 82,4 & 68,0 & $77,7 \mathrm{ab}$ \\
\hline $\mathrm{GA}_{3}\left(100 \mathrm{mg} \mathrm{L}^{-1}\right)$ & 84,9 & 91,3 & 63,8 & $80,0 \mathrm{ab}$ \\
\hline $\mathrm{GA}_{3}\left(100 \mathrm{mg} \mathrm{L}^{-1}\right)+\operatorname{AVG}\left(90 \mathrm{mg} \mathrm{L}^{-1}\right)$ & 84,2 & 87,2 & 70,2 & $80,5 \mathrm{ab}$ \\
\hline $\mathrm{GA}_{3}\left(100 \mathrm{mg} \mathrm{L}^{-1}\right)+\operatorname{AVG}\left(125 \mathrm{mg} \mathrm{L}^{-1}\right)$ & 83,8 & 89,3 & 73,2 & $82,1 \mathrm{a}$ \\
\hline Média & $83,0 \mathrm{~A}$ & $85,1 \mathrm{~A}$ & $68,6 \mathrm{~B}$ & \\
\hline \multirow[t]{2}{*}{ CV (\%) } & & 10,09 & & \\
\hline & \multicolumn{3}{|c|}{ Acidez Titulável (meq 100 $\left.\mathrm{mL}^{-1}\right)$} & Média \\
\hline Controle & 31,1 & 33,4 & 29,5 & $31,3 \mathrm{a}$ \\
\hline $\operatorname{AVG}\left(90 \mathrm{mg} \mathrm{L}^{-1}\right)$ & 35,6 & 33,0 & 26,2 & $31,6 a$ \\
\hline $\operatorname{AVG}\left(125 \mathrm{mg} \mathrm{L}^{-1}\right)$ & 35,2 & 32,1 & 24,6 & $30,6 \mathrm{a}$ \\
\hline $\mathrm{GA}_{3}\left(100 \mathrm{mg} \mathrm{L}^{-1}\right)$ & 32,8 & 30,6 & 23,7 & $29,0 \mathrm{a}$ \\
\hline $\mathrm{GA}_{3}^{3}\left(100 \mathrm{mg} \mathrm{L}^{-1}\right)+\operatorname{AVG}\left(90 \mathrm{mg} \mathrm{L}^{-1}\right)$ & 30,4 & 30,6 & 24,5 & $28,5 \mathrm{a}$ \\
\hline $\mathrm{GA}_{3}\left(100 \mathrm{mg} \mathrm{L}^{-1}\right)+\operatorname{AVG}\left(125 \mathrm{mg} \mathrm{L}^{-1}\right)$ & 33,0 & 30,7 & 23,5 & $29,0 \mathrm{a}$ \\
\hline Média & $33,0 \mathrm{~A}$ & $31,7 \mathrm{~A}$ & $25,3 \mathrm{~B}$ & \\
\hline \multirow[t]{2}{*}{ CV (\%) } & & 4,83 & & \\
\hline & \multicolumn{3}{|c|}{ Sólidos Solúveis Totais $\left({ }^{\circ}\right.$ Brix $)$} & Média \\
\hline Controle & 10,3 & 10,4 & 10,2 & $10,3 \mathrm{a}$ \\
\hline AVG $\left(90 \mathrm{mg} \mathrm{L}^{-1}\right)$ & 9,6 & 10,0 & 9,3 & $9,6 \mathrm{~b}$ \\
\hline $\operatorname{AVG}\left(125 \mathrm{mg} \mathrm{L}^{-1}\right)$ & 9,9 & 10,1 & 9,0 & $9,7 \mathrm{~b}$ \\
\hline $\mathrm{GA}_{3}\left(100 \mathrm{mg} \mathrm{L}^{-1}\right)$ & 9,6 & 8,7 & 7,7 & $8,7 \mathrm{c}$ \\
\hline $\mathrm{GA}_{3}\left(100 \mathrm{mg} \mathrm{L}^{-1}\right)+\operatorname{AVG}\left(90 \mathrm{mg} \mathrm{L}^{-1}\right)$ & 9,4 & 9,1 & 7,7 & $8,7 \mathrm{c}$ \\
\hline $\mathrm{GA}_{3}\left(100 \mathrm{mg} \mathrm{L}^{-1}\right)+\operatorname{AVG}\left(125 \mathrm{mg} \mathrm{L}^{-1}\right)$ & 9,3 & 8,9 & 8,1 & $8,8 \mathrm{c}$ \\
\hline Média & $9,7 \mathrm{~A}$ & $9,5 \mathrm{~A}$ & $8,7 \mathrm{~B}$ & \\
\hline $\mathrm{CV}(\%)$ & & 6,34 & & \\
\hline
\end{tabular}

* Médias seguidas por mesma letra, maiúscula na horizontal e minúscula na vertical, não diferem entre si, pelo teste de Tukey (p<0,05). 
TABELA 4 - Cor da epiderme, acidez titulável e sólidos solúveis em ameixas 'Laetitia', na colheita, em função da aplicação pré-colheita de aminoetoxivinilglicina (AVG) e ácido giberélico (GA $\left.{ }_{3}\right)$ e data de colheita, no ano de 2009.

\begin{tabular}{|c|c|c|c|c|}
\hline \multirow[b]{2}{*}{ Tratamentos } & \multicolumn{3}{|c|}{$2008 / 2009$} & \multirow[b]{2}{*}{ Média } \\
\hline & $1^{\text {a }}$ Colheita & $2^{\text {a }}$ Colheita & $3^{\text {a }}$ Colheita & \\
\hline \multicolumn{5}{|c|}{ Cor (hue) } \\
\hline Controle & 76,6 & 72,2 & 64,3 & $71,0 \mathrm{c}^{*}$ \\
\hline $\operatorname{AVG}\left(90 \mathrm{mg} \mathrm{L}^{-1}\right)$ & 75,5 & 78,2 & 63,9 & $72,5 \mathrm{bc}$ \\
\hline AVG (125 mg L-1) & 80,6 & 80,3 & 66,3 & $75,8 b$ \\
\hline $\mathrm{GA}_{3}\left(100 \mathrm{mg} \mathrm{L}^{-1}\right)$ & 91,7 & 98,6 & 68,9 & $86,4 \mathrm{a}$ \\
\hline $\mathrm{GA}_{3}\left(100 \mathrm{mg} \mathrm{L}^{-1}\right)+\operatorname{AVG}\left(90 \mathrm{mg} \mathrm{L}^{-1}\right)$ & 85,0 & 88,1 & 70,9 & $81,3 \mathrm{a}$ \\
\hline $\mathrm{GA}_{3}\left(100 \mathrm{mg} \mathrm{L}^{-1}\right)+\operatorname{AVG}\left(125 \mathrm{mg} \mathrm{L}^{-1}\right)$ & 91,3 & 97,3 & 79,8 & $89,5 \mathrm{a}$ \\
\hline Média & $83,5 \mathrm{~A}$ & $85,8 \mathrm{~A}$ & $69,0 \mathrm{~B}$ & \\
\hline \multirow[t]{2}{*}{$\mathrm{CV}(\%)$} & & 9,31 & & \\
\hline & \multicolumn{3}{|c|}{ 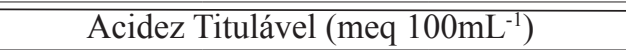 } & Média \\
\hline Controle & 31,7 & 33,8 & 30,5 & $30,7 \mathrm{a}$ \\
\hline $\operatorname{AVG}\left(90 \mathrm{mg} \mathrm{L}^{-1}\right)$ & 35,0 & 32,9 & 26,0 & $31,3 \mathrm{a}$ \\
\hline $\operatorname{AVG}\left(125 \mathrm{mg} \mathrm{L}^{-1}\right)$ & 34,9 & 31,8 & 24,3 & $30,3 \mathrm{a}$ \\
\hline $\mathrm{GA}_{3}\left(100 \mathrm{mg} \mathrm{L}^{-1}\right)$ & 33,0 & 30,8 & 23,9 & $29,2 \mathrm{a}$ \\
\hline $\mathrm{GA}_{3}\left(100 \mathrm{mg} \mathrm{L}^{-1}\right)+\operatorname{AVG}\left(90 \mathrm{mg} \mathrm{L}^{-1}\right)$ & 31,4 & 31,6 & 25,5 & $29,5 \mathrm{a}$ \\
\hline $\mathrm{GA}_{3}\left(100 \mathrm{mg} \mathrm{L}^{-1}\right)+\operatorname{AVG}\left(125 \mathrm{mg} \mathrm{L}^{-1}\right)$ & 32,9 & 30,5 & 24,5 & $29,3 \mathrm{a}$ \\
\hline Média & $33,2 \mathrm{~A}$ & $31,9 \mathrm{~A}$ & $25,8 \mathrm{~B}$ & \\
\hline \multirow[t]{2}{*}{ CV $(\%)$} & & 6,54 & & \\
\hline & \multicolumn{3}{|c|}{ Sólidos Solúveis Totais ( $\left.{ }^{\circ} \mathrm{Brix}\right)$} & Média \\
\hline Controle & 10,1 & 10,2 & 10,0 & $10,1 \mathrm{a}$ \\
\hline AVG (90 mg L-1) & 9,5 & 9,8 & 9,8 & $9,7 \mathrm{ab}$ \\
\hline $\operatorname{AVG}\left(125 \mathrm{mg} \mathrm{L}^{-1}\right)$ & 9,7 & 9,8 & 9,4 & $9,6 b$ \\
\hline $\mathrm{GA}_{3}\left(100 \mathrm{mg} \mathrm{L}^{-1}\right)$ & 9,8 & 9,4 & 8,3 & $9,2 \mathrm{bc}$ \\
\hline $\mathrm{GA}_{3}\left(100 \mathrm{mg} \mathrm{L}^{-1}\right)+\operatorname{AVG}\left(90 \mathrm{mg} \mathrm{L}^{-1}\right)$ & 9,5 & 9,2 & 7,8 & $8,8 \mathrm{c}$ \\
\hline $\mathrm{GA}_{3}\left(100 \mathrm{mg} \mathrm{L}^{-1}\right)+\operatorname{AVG}\left(125 \mathrm{mg} \mathrm{L}^{-1}\right)$ & 9,5 & 8,7 & 8,3 & $8,8 \mathrm{c}$ \\
\hline Média & $9,7 \mathrm{~A}$ & $9,5 \mathrm{~A}$ & $8,9 \mathrm{~B}$ & \\
\hline CV $(\%)$ & & 6,76 & & \\
\hline
\end{tabular}

"Médias seguidas por mesma letra, maiúscula na horizontal e minúscula na vertical, não diferem entre si, pelo teste de Tukey (p<0,05). 
TABELA 5 -Firmeza de polpa e forças para a penetração da polpa e para compressão do fruto e teor de SS de ameixas 'Laetitia' tratadas em pré-colheita com AVG e GA 3 e armazenadas por 22 dias a $1^{\circ} \mathrm{C}$ e $92 \%$ de UR.

\begin{tabular}{lcccc}
\hline \multicolumn{1}{c}{ Tratamentos } & $\begin{array}{c}\text { Firmeza de } \\
\text { polpa (N) }\end{array}$ & \multicolumn{2}{c}{ Atributos de textura (N) } & Força para a \\
penetração da polpa & $\begin{array}{c}\text { Força para a } \\
\text { compressão do fruto }\end{array}$ & SS ('Brix) \\
\hline Controle & $15,8 \mathrm{c}^{*}$ & $0,85 \mathrm{c}$ & $20,1 \mathrm{~b}$ & $10,3 \mathrm{a}$ \\
& & & & \\
& & & & \\
& & & & \\
$\mathrm{AVG}\left(90 \mathrm{mg} \mathrm{L}^{-1}\right)$ & $22,5 \mathrm{bc}$ & $1,21 \mathrm{bc}$ & $25,7 \mathrm{ab}$ & $9,7 \mathrm{ab}$ \\
$\mathrm{AVG}\left(125 \mathrm{~m} \mathrm{~L}^{-1}\right)$ & $24,4 \mathrm{bc}$ & $1,04 \mathrm{bc}$ & $28,0 \mathrm{ab}$ & $9,1 \mathrm{bc}$ \\
$\mathrm{GA}_{3}\left(100 \mathrm{mg} \mathrm{L}^{-1}\right)$ & $19,9 \mathrm{c}$ & $0,88 \mathrm{bc}$ & $27,7 \mathrm{ab}$ & $9,1 \mathrm{bc}$ \\
$\mathrm{GA}_{3}\left(100 \mathrm{mg} \mathrm{L}^{-1}\right)+\mathrm{AVG}\left(90 \mathrm{mg} \mathrm{L}^{-1}\right)$ & $34,0 \mathrm{a}$ & $1,66 \mathrm{a}$ & $35,2 \mathrm{a}$ & $8,7 \mathrm{c}$ \\
$\mathrm{GA}_{3}\left(100 \mathrm{mg} \mathrm{L}^{-1}\right)+\mathrm{AVG}\left(125 \mathrm{mg} \mathrm{L}^{-1}\right)$ & $30,8 \mathrm{ab}$ & $1,27 \mathrm{ab}$ & $33,8 \mathrm{a}$ & $8,7 \mathrm{c}$ \\
$\mathrm{CV}(\%)$ & 12,7 & 12,5 & 13,9 & 3,6 \\
\hline
\end{tabular}

${ }^{*}$ Médias seguidas por mesma letra na vertical não diferem entre si, pelo teste de Tukey $(\mathrm{p}<0,05)$.

\section{CONCLUSÕES}

1-A aplicação de AVG retarda a maturação da ameixa 'Laetitia' na dose de $125 \mathrm{mg} \mathrm{L}^{-1}$.

2-A aplicação pré-colheita de $\mathrm{GA}_{3}$, seguido ou não da aplicação com AVG, apresenta melhores resultados no retardo da maturação de ameixas 'Laetitia'.

3-A aplicação pré-colheita de $\mathrm{GA}_{3}$, seguida da aplicação com AVG, possibilita maior firmeza da polpa de ameixas cultivar Laetitia armazenadas sob refrigeração.

\section{AGRADECIMENTOS}

Agradecemos à empresa Fruticultura Pilati, pela parceria neste trabalho, que forneceu a área para realização deste experimento. Também ao $\mathrm{CNPq}$ e à UDESC, pela concessão das Bolsas de Iniciação Científica e de Produtividade em Pesquisa.

\section{REFERÊNCIAS}

AMARANTE, C.V.T. do; DREHMER, M.M.F.; SOUZA, F.; FRANCESCATO, P. A pulverização pré-colheita com ácido giberélico $\left(\mathrm{GA}_{3}\right)$ e aminoetoxivinilglicina (AVG) retarda a maturação e reduz as perdas de frutos na cultura do pessegueiro. Revista Brasileira de Fruticultura, Jaboticabal, v.27, n.1, p.1-5, 2005.

AMARANTE, C.V.T. do; STEFFENS, C.A. O tratamento pré-colheita com AVG, aliado à absorção de etileno durante o armazenamento refrigerado, preserva a qualidade de maçãs 'Gala'. Revista Brasileira de Fruticultura, Jaboticabal, v.31, n.2, p.334-342, 2009.

ALVES, E.O.; STEFFENS, C.A.; AMARANTE, C.V.T.; PAVANELLO, E.P.; BRACKMANN, A. Manejo do etileno durante o armazenamento de ameixas 'Laetitia' em atmosfera controlada. Ciência Rural, Santa Maria, v.39, n.9, p.2445-245, 2009a.

ALVES, E.O.; STEFFENS, C.A.; AMARANTE, 
C.V.T.; WEBER, A.; MIQUELOTO, A.; BRACKMANN, A. Armazenamento refrigerado de ameixas 'Laetitia' com uso de 1-MCP e indução de perda de massa fresca. Ciência Rural, Santa Maria, v.40, n.1, p.30-36, 2010.

BEN-ARIE, R.; SAKS, Y.; SONEGO, L.; FRANK, A. Cell wall metabolism in giberillin-treated persimmon fruits. Plant Growth Regulation, Dordrecht, v.19, n.1, p.25-33, 1996.

BRACKMANN, A.; BENEDETTI, M.; HUNSCHE, M.; SESTARI, I. Armazenamento de ameixas cvs. Reubennel e Pluma 7 sob diferentes temperaturas, em atmosfera controlada e refrigerada. Revista Brasileira de Agrociência, Pelotas, v.11, n.1, p.8589, 2005.

BRACKMANN, A.; MELLO, A.M.; FREITAS, S.T. Qualidade pós-colheita de caqui 'Kyoto', tratados com ácido giberélico e aminoetoxivinilglicina em pré-colheita. Revista da Faculdade de Zootecnia, Veterinária e Agronomia, Uruguaiana, v.9, n.1, p.48-55, 2002.

CANLI, F.A.; ORHAN, H. Effects of preharvest gibberellic acid applications on fruit quality of '0900 Zirrat' sweet cherry. HortTechnology, Alexandria, v.19, n.1, p.127-129, 2009.

FERRI, V.C.; RINALDI, M.M.; SILVA, J.A.; LU-
CHETTA, L.; MARINI, L.; ROMBALDI, C.V. Ácido giberélico no retardamento da maturação de caquis (Diospyrus kaki, L.) cultivar Fuyu. Ciência e Tecnologia dos Alimentos, Campinas, v.24, n.1, p.1-5, 2004.

GUILLERMIN, P.; DUPONT, N.; LE MORVAN, C.; LE QUÉRÉ, J.-M.; LANGLAIS, C.; MAUGET, J.C. Rheological and technological properties of two cider apple cultivars. LWT - Food Science and Technology, Amsterdam, v. 39, n.9, p. 995-1000, 2006.

JU, Z.; DUAN, Y.; JU, Z. Combinations of GA3 and AVG delay fruit maturation, increase fruit size and improve storage life of 'Feicheng' peaches. Journal of Horticultural Science \& Biotechnology, Asford, v.74, n.5, p.579-583, 1999.

MAJUMDER, K.; MAZUMDAR, B.C. Changes of pectic substances in developing fruits of capegooseberry (Physalis peruviana L.) in relation to the enzyme activity and evolution of ethylene. Scientia Horticulturae, Amsterdam, v.96, n.1-4, p.91-101, 2002.

PECH, J.C.; LATCHÉ, A.; BALAGUÉ, C.; BOUZAYEN, M.; LELIÈVRE, J.M. Postharvest physiology of climacteric fruits: recent development in the biosynthesis and action of ethylene. Sciencia Alimentaria, Toulouse, v.14, n.1, p.3-14, 1994.

STEFFENS, C.A.; AMARANTE, C.V.T. do; CHECHI, R.; SILVEIRA, J.P.G.; BRACKMANN, A. Aplicação pré-colheita de reguladores vegetais visando a retardar a maturação de ameixas 'Laetitia'. Ciência Rural, Santa Maria, v.39, n.5, ago. 2009.

STEFFENS, C.A.; GIEHL, R.F.H.; BRACKMANN, A. Maçã 'Gala' armazenada em atmosfera controlada e tratada com aminoetovinilglicina e ethephon. Pesquisa Agropecuária Brasileira, Brasília, v.40, n.9, p.837-843, 2005.

STEFFENS, C.A.; GUARIENTI, A.J.W.; STORCK, L.; BRACKMANN, A. Maturação da maçã 'Gala' com a aplicação pré-colheita de aminoetoxivinilglicina e ethephon. Ciência Rural, Santa Maria, v.36, n.2, p. 434-440, 2006. 